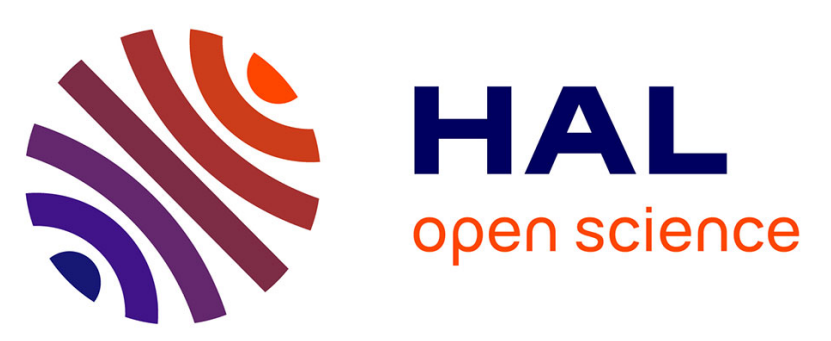

\title{
Optical flow-based controller for reactive and relative navigation dedicated to a four rotor rotorcraft
}

\author{
Eduardo Rondon, Isabelle Fantoni-Coichot, Anand Sanchez, Guillaume
}

Sanahuja

\section{- To cite this version:}

Eduardo Rondon, Isabelle Fantoni-Coichot, Anand Sanchez, Guillaume Sanahuja. Optical flow-based controller for reactive and relative navigation dedicated to a four rotor rotorcraft. IEEE/RSJ International Conference on Intelligent Robots and Systems, IROS 2009, Oct 2009, Saint Louis, United States. pp.684-689. hal-00445997

\section{HAL Id: hal-00445997 https://hal.science/hal-00445997}

Submitted on 11 Jan 2010

HAL is a multi-disciplinary open access archive for the deposit and dissemination of scientific research documents, whether they are published or not. The documents may come from teaching and research institutions in France or abroad, or from public or private research centers.
L'archive ouverte pluridisciplinaire HAL, est destinée au dépôt et à la diffusion de documents scientifiques de niveau recherche, publiés ou non, émanant des établissements d'enseignement et de recherche français ou étrangers, des laboratoires publics ou privés. 


\title{
Optical Flow-Based Controller for Reactive and Relative Navigation dedicated to a Four Rotor Rotorcraft
}

\author{
Eduardo Rondon, Isabelle Fantoni-Coichot, Anand Sanchez, Guillaume Sanahuja
}

\begin{abstract}
Autonomous navigation of an unmanned aerial vehicle (UAV) can be achieved with a reactive system which allows the robot to overcome all the unexpected changes in its environment. In this article, we propose a new approach to avoid frontal obstacles using known properties of the optical flow and by taking advantage of the capability of stationary flight of the rotorcraft. A state machine is proposed as a solution to equip the UAV with all reactions necessary for indoor navigation. We show how smooth transitions can be achieved by decreasing the speed of the vehicle proportional to the distance to an obstacle and by brief instants of hovering flight. Each stage of our algorithm has been tested in a mobile robot.
\end{abstract}

\section{INTRODUCTION}

In recent years, there has been a growing interest on the development of unmanned aerial vehicles. The capability to fly indoor as well as outdoor makes this kind of autonomous systems very suitable for delicate applications such traffic monitoring, search and rescue missions and surveillance operations. Many efforts have been done in the development of these autonomous aerial vehicles, and at present realtime embedded stabilization has been already proved [1] [2]. However, for a complete implementation of UAVs on real life applications, the robot must be capable to react against unexpected changes of its environment, and to navigate through unknown environments. Moreover if one wants to guide unmanned vehicles one must also have a selfcontained navigation system, which means that one must find a way to keep track of position, and speed continuously. The Global Positioning System is widely used to assure the self-position task, but GPS signals are not available in all locations. Indeed, this system can not be used for indoor flights and the well known canyon problem can occur in urban environment where the signal is continuously blocked by high-rise buildings.

Image-based navigation arises as a complementary system for the GPS. The main advantage of image-based navigation is that it provides detailed information about the environment of the moving object. Due to this interaction between the sensor with its surrounding, appropriate techniques may be developed permitting the autonomous vehicle to find their way even within unknown environment. Moreover, optical flow which is the estimation of the motion field created by a moving camera with respect to a rigid scene, plays a more

This work was supported by the French Research Foundation for Aeronautics and Space

E. Rondon, I. Fantoni-Coichot, A. Sanchez and G. Sanahuja are with the Université de Technologie de Compiègne - CNRS UMR 6599 Heudiasyc, BP 20529, 60205 Compiègne Cedex, France Eduardo.Rondonehds.utc. fr and more important role in the development of robotics. In the literature, optical flow is widely used for the design of navigation algorithms [3], obstacle avoidance [4] and robot stabilization [5]. Furthermore, because translational optical flow is inversely proportional to the distance between the image plane and the surrounding objets, it can be used as a notion of relative positioning. Herisse et al. [6] have on this subject developed an optical flow based landing control. Serres et al. [7] demonstrated an approach that allows an autonomous vehicle to move at the center of a corridor. In [8] the author established a relation between the $\mathrm{OF}$ $\left(\frac{\text { pixel }}{s}\right)$ and the actual rotation $\left(\frac{\text { degrees }}{s}\right)$. After a calibration, the cancelation of the rotational components is obtained by substracting the gyroscopic value from the OF measurement. They use this fusion system in [9] to avoid the obstacles presents on a microflyer path.In this paper, we propose a state machine approach for the relative and reactive navigation of a rotorcraft.

Two orthogonal cameras have been used in this study to construct the optical flow-based controllers. When moving in the forward direction, the lateral velocity is stabilized at zero and the forward speed is set to a constant value. In the opposite case, when moving in the lateral direction, the lateral velocity is set to a constant value, while the forward velocity is stabilized at zero. In both cases, an anti-collision controller acts as a preventive system. This system measures the inverse of the time-to-contact and decelerates the vehicle proportionally to this measure. Once the obstacle is too close the vehicle stops and another controller is activated. We describe how smooth transitions between states can be achieved with our approach. A new avoiding controller is proposed which uses the moving average of the optical flow to automatically stop the vehicle once the obstacle is overtaken. This controller can be used for both, lateral avoidance and altitude avoidance. After a short time of hovering flight, the state machine starts again the forward motion. The overall system includes speed control, anticollision system, and lateral and altitude obstacle avoidance. This system is ready to be coupled with urban canyon and wall following tasks.

The remainder of this paper is organized as follows. In Section 2, we describe the method used for an appropriate implementation of the optical flow. Section 3 specifies the dynamical model of the helicopter. In section 4 the proposed control law is discussed. In Section 5, we show some experimental results. We conclude our work in Section 6. 


\section{VISUAL SYSTEM}

The robot is equipped with two orthogonal cameras, both cameras having a wide field of view optics. These cameras are placed orthogonally, the frontal camera $\left(\mathrm{cam}_{f}\right)$ and the downward camera $\left(\mathrm{cam}_{d}\right)$ (see Figures 1 and 2). The goal is to control the speed of the engine with $\mathrm{cam}_{d}$ and to detect and avoid obstacles with $\operatorname{cam}_{f}$. The system is described below:

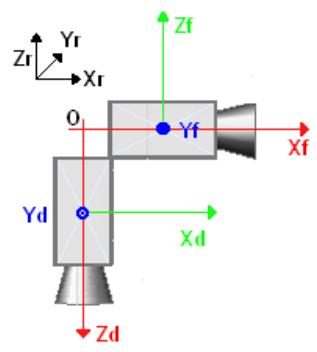

Fig. 1. Visual perspective of the cameras

- rotorcraft coordinate frame:

To simplify the notation, the point $O$ (intersection between the two optical axis $X_{f}$ and $Z_{d}$ ) is used as the center of the rotorcraft coordinate frame. The pitch, roll and yaw axes are noted $X_{r}, Y_{r}$ and $Z_{r}$ respectively.

- cam $_{f}$ image plane:

The optical axis of the camera $X_{f}$ is collinear with the roll axis of the UAV. The corresponding image plane $\left(Y_{f}, Z_{f}\right)$ is parallel with the plane formed by $\left(Y_{r}, Z_{r}\right)$. The coordinates of the optical flow, computed in this image plane, are noted $O F_{y}^{f}$ and $O F_{z}^{f}$.

- cam $_{d}$ image plane:

The optical axis $Z_{d}$ is collinear with the yaw axis of the helicopter. The image plane of the camera $\left(X_{d}, Y_{d}\right)$ is parallel with the plane form by $\left(X_{r},-Y_{r}\right)$. The coordinates of the optical flow, computed in this image plane, are noted $O F_{x}^{d}$ and $O F_{y}^{d}$.

From the optical flow obtained by $\mathrm{cam}_{d}$, an information of the forward and lateral speed of the engine can be extracted. This information is used to control the displacement of the vehicle in the pitch and roll axis. Once the vehicle travels at a constant speed, the optical flow obtained by cam $_{f}$ gives an information of the closeness to the objects in the scene, and it is used to design the detection and avoidance subsystem.

\section{A. Computing Optical Flow}

In this study, the Lucas-Kanade pyramidal algorithm [10] has been implemented to make the calculations efficient and to allow large movements of the robot. For $\operatorname{cam}_{d}$, a rich texture detector is used in order to calculate the velocity vector in the travel movement. However for $\operatorname{cam}_{f}$, a predefined mesh of points is used and the optical flow is repeatedly computed on this mesh in order to detect an obstacle. It is well known that computing the optical flow in the robot environment still presents many issues. Occlusion, bad illumination and bad texture images can lead to noisy optical flow estimates. Also, moving objects in the scene and robot self rotations give a biased motion field. To compensate the rotational components of the optical flow and to filter the measures, a novel fusion approach between the Inertial Measurement Unit (IMU) and the optical flow is implemented. This approach is based on Kalman Filter theory. Our fusion method takes into advantage known properties of the optical flow like the approach proposed by Romero et al.[11], but it differs by the fact that our Kalman Filter approach is an optimal solution for incrementally reducing the uncertainty of the desired translational velocities, since we incorporate representations of the uncertainty of the Optical Flow model and of the inertial measurements. Muratet et al. [12] use a fusion method which needs the computation of the vector $R_{O F}$ (rotational component) in each point having an estimated optical flow. This means the computation of $N$ matrix products, where $N$ is the total number of points. Our approach, on the contrary, only needs to compute on each step the discrete Kalman Filter equations. In the following, the algorithm will be detailed for $\mathrm{cam}_{d}$. Considering the rotorcraft as a rigid body moving in a $3 \mathrm{D}$ space, the six motion parameters can be related to the image centered coordinate frame.

$$
\left[\begin{array}{c}
O F_{x_{i}}^{d} \\
O F_{y_{i}}^{d}
\end{array}\right]=T_{O F}^{d}+R_{O F}^{d}
$$

with the translational part

$$
T_{O F}^{d}=\frac{1}{Z}\left[\begin{array}{ccc}
-f & 0 & x_{i} \\
0 & -f & y_{i}
\end{array}\right]\left[\begin{array}{c}
V_{\mathrm{x}} \\
V_{\mathrm{y}} \\
V_{\mathrm{z}}
\end{array}\right]
$$

and the rotational part

$$
R_{O F}^{d}=\left[\begin{array}{ccc}
\frac{x_{i} y_{i}}{f} & -\left(f+\frac{x_{i}^{2}}{f}\right) & y_{i} \\
\left(f+\frac{y_{i}^{2}}{f}\right) & -\frac{x_{i} y_{i}}{f} & -x_{i}
\end{array}\right]\left[\begin{array}{c}
\omega_{\mathrm{x}} \\
\omega_{\mathrm{y}} \\
\omega_{\mathrm{z}}
\end{array}\right]
$$

where $O F_{j_{i}}^{d}$ is the optical flow component in the coordinate $j$ of the point $p_{i}\left(x_{i}, y_{i}\right), V_{\mathrm{k}}$ and $\omega_{\mathrm{k}}$ are the translation velocities and rotation rates, respectively, of the body in the coordinate $\mathrm{k}$ and $f$ is the focal distance. When computing the optical flow, all the points $p_{i}$ are known. Furthermore, they all share the same movement, we can express the mean of the optical flow as follows

$$
\begin{aligned}
& O \bar{F}_{x}^{d}=\bar{V}_{O F_{\mathrm{x}}}^{d}+K_{x}^{x} \bar{V}_{O F_{\mathrm{z}}}^{d}+K_{x y}^{x} \omega_{\mathrm{x}}-K_{x^{2}}^{x} \omega_{\mathrm{y}}+K_{y}^{x} \omega_{\mathrm{z}} \\
& O \bar{F}_{y}^{d}=\bar{V}_{O F_{\mathrm{y}}}^{d}+K_{y}^{y} \bar{V}_{O F_{\mathrm{z}}} v+K_{y^{2}}^{y} \omega_{\mathrm{x}}-K_{x y}^{y} \omega_{\mathrm{y}}-K_{x}^{y} \omega_{\mathrm{z}}
\end{aligned}
$$

with

$$
\begin{array}{r}
\bar{V}_{O F_{\mathrm{x}}}^{d}=-f \frac{V_{\mathrm{x}}}{Z} \\
\bar{V}_{O F_{\mathrm{y}}}^{d}=-f \frac{V_{\mathrm{y}}}{Z} \\
\bar{V}_{O F_{\mathrm{z}}}^{d}=\frac{V_{\mathrm{z}}}{Z}
\end{array}
$$

where $O \bar{F}_{x}^{d}$ and $O \bar{F}_{y}^{d}$ are the means of the sensed optical flow in the image coordinate system. $\bar{V}_{O F_{\mathrm{x}}}^{d}, \bar{V}_{O F_{\mathrm{y}}}^{d}$ are the relative velocities of the aircraft in the camera coordinate system. $\bar{V}_{O F_{\mathrm{Z}}}^{d}$ is the inverse of the time-to-contact, known 
as the relative depth, and $K j^{i}$ are the constant scale factors depending on intrinsic parameters of the camera. These parameters can be computed separately and allow us to know the contribution of the rotorcraft rotations to the optical flow. A discrete time Kalman filter is designed to compensate the rotational motion field.

$$
\left[\begin{array}{c}
\bar{V}_{O F} \\
W
\end{array}\right]_{k}=\left[\begin{array}{ll}
I & 0 \\
0 & I
\end{array}\right]\left[\begin{array}{c}
\bar{V}_{O F} \\
W
\end{array}\right]_{k-1}+\left[\begin{array}{l}
v_{1} \\
v_{2}
\end{array}\right]_{k}
$$

Here $\bar{V}_{O F}$ is the vector composed of the mean translational components of the optical flow, and $W$ is the vector composed of angular velocities and $v_{i}$ represents the noise.

$$
\begin{aligned}
\bar{V}_{O F} & =\left[\bar{V}_{O F_{\mathrm{x}}}^{d}+K_{x}^{x} \bar{V}_{O F_{\mathrm{z}}}^{d}, \bar{V}_{O F_{\mathrm{y}}}^{d}+K_{y}^{y} \bar{V}_{O F_{\mathrm{z}}}^{d}\right]^{T} \\
& =\left[\tilde{V}_{O F_{\mathrm{x}}}, \tilde{V}_{O F_{\mathrm{y}}}\right]^{T}
\end{aligned}
$$

The measured outputs are the optical flow means $O \bar{F}^{d}$, and the measured angular velocity $W_{I M U}$. The outputs are related to the state in $(8)$ as

$$
\left[\begin{array}{c}
O \bar{F}^{d} \\
W_{I M U}
\end{array}\right]_{k}=\left[\begin{array}{cc}
I & K_{R}^{T} \\
0 & I
\end{array}\right]\left[\begin{array}{c}
\bar{V}_{O F} \\
W
\end{array}\right]_{k}+\left[\begin{array}{c}
v_{1} \\
v_{2}
\end{array}\right]_{k}
$$

where the noises in (8) and (11) are:

- $v=\left[v_{1}, v_{2}\right]^{T}$ is assumed to be a white noise with zero mean and known constant covariance matrix $Q$.

- $v=\left[v_{1}, v_{2}\right]^{T}$ is assumed to be a white noise with zero mean and known constant covariance matrix $R$.

- $v$ and $v$ are assumed to be uncorrelated noises.

To compute the optical flow noise covariance matrix we take advantage of the properties of the model. Because the robot is modeled as a rigid body, the standard deviation between the spatial mean of the optical flow (eg. $\left.\tilde{V}_{O F_{\mathrm{x}}}\right)$ and the optical flow in each point (eg. $O F_{x_{i}}^{d}$ ) is bounded. Any point having an optical flow too distant of the mean will be ignored. At the end, the reliability of the optic flow is defined as a function of the desired constant bound $\left(\sigma_{i}^{o}\right)$, the total number of points having an estimate $\left(N_{d}\right)$ and the wrong measurements $\left(n_{d}\right)$.

$$
R=\left[\begin{array}{cc}
f\left(\sigma_{i}^{o}, n_{d}, N_{d}\right) & 0 \\
0 & R_{\text {imu }}
\end{array}\right]
$$

After the filter step, the three translational velocities can be estimated up to a scale factor, by means of a simplified egomotion algorithm [13]. From the Kalman Filter we have

$$
\begin{aligned}
\left\|O F_{x_{i}}^{d}-\left(K_{x y}^{x} \omega_{\mathrm{x}}-K_{x^{2}}^{x} \omega_{\mathrm{y}}+K_{y}^{x} \omega_{\mathrm{z}}\right)\right\| & \leq \sigma_{i}^{o} \tilde{V}_{O F_{\mathrm{x}}} \\
\left\|O F_{y_{i}}^{d}-\left(K_{y^{2}}^{y} \omega_{\mathrm{x}}-K_{x y}^{y} \omega_{\mathrm{y}}-K_{x}^{y} \omega_{\mathrm{z}}\right)\right\| & \leq \sigma_{i}^{o} \tilde{V}_{O F_{\mathrm{y}}}
\end{aligned}
$$

The $N_{d}-n_{d}$ points satisfying (13) and (14) can be written as

$$
\left[\begin{array}{c}
O F_{x_{i}}^{d} \\
O F_{y_{i}}^{d}
\end{array}\right]=\bar{V}_{O F_{\mathrm{z}}}^{d}\left[\begin{array}{ll}
1 & 0 \\
0 & 1
\end{array}\right]\left[\begin{array}{c}
x_{i} \\
y_{i}
\end{array}\right]+\left[\begin{array}{c}
\bar{V}_{O F_{\mathrm{x}}}^{d} \\
\bar{V}_{O F_{\mathrm{y}}}^{d}
\end{array}\right]
$$

Here $\bar{V}_{O F_{\mathrm{z}}}$ forms a virtual scaling factor $c$, the identity matrix forms a virtual rotation matrix $R$, and $\bar{V}_{O F_{\mathrm{x}}}$ and $\bar{V}_{O F_{\mathrm{y}}}$ a virtual translation vector $t$. We can estimate the three translational components of the optical flow by minimizing
$\left\|O F_{i}^{d}-(c R \vec{x}+t)\right\|^{2}$ in a least square approach. The solution of this problem is based on a singular value decomposition of the covariance matrix of the data (for details see [13]).

\section{B. Obstacle Detection and Avoidance using optical flow}

1) Time-To-Contact: In order to allow our algorithm to detect an obstacle, we use the estimated velocities to compute the time-to-contact. In the $\left(Y_{f}, Z_{f}\right)$ image plane and using the camera $\operatorname{cam}_{f}$, these velocities can be written as

$$
\begin{gathered}
O F_{y_{i}}^{f}=\bar{V}_{O F_{\mathrm{y}}}^{f}+y_{i} \bar{V}_{O F_{\mathrm{x}}}^{f} \\
O F_{z_{i}}^{f}=\bar{V}_{O F_{\mathrm{z}}}^{f}+z_{i} \bar{V}_{O F_{\mathrm{x}}}^{f}
\end{gathered}
$$

where $x_{i}$ and $y_{i}$ are the coordinates of points where optical flow is computed. These points are constant in the image mesh used to compute the motion field. By taking into account that the focus of expansion is the point where optical flow converges we have

$$
\begin{gathered}
0=\bar{V}_{O F_{\mathrm{y}}}^{f}+y_{f o e} \bar{V}_{O F_{\mathrm{x}}}^{f} \\
0=\bar{V}_{O F_{\mathrm{z}}}^{f}+z_{f o e} \bar{V}_{O F_{\mathrm{x}}}^{f}
\end{gathered}
$$

Then, by using (16) and (17), in each point $\left(x_{i}, y_{i}\right)$, there is

$$
\begin{gathered}
O F_{y_{i}}^{f}=\bar{V}_{O F_{\mathrm{x}}}^{f}\left(y_{i}-y_{f o e}\right) \\
O F_{z_{i}}^{f}=\bar{V}_{O F_{\mathrm{x}}}^{f}\left(z_{i}-z_{f o e}\right)
\end{gathered}
$$

By taking the mean of the optical flow on all the points which are the outputs of the Kalman Filter, we can compute the inverse of the time-to-contact as follows

$$
\eta=\bar{V}_{O F_{\mathrm{x}}}=\frac{\sqrt{\tilde{V}_{O F_{\mathrm{y}}}^{2}+\tilde{V}_{O F_{\mathrm{z}}}^{2}}}{K_{\eta}}
$$

Here $K_{\eta}$ is a constant specific to $\operatorname{cam}_{f}$, that depends on the parameters $K_{y}^{y}$ and $K_{z}^{z}$ computed previously in the Kalman Filter, and on the focus of expansion.

The function $\eta(t)$ depends on time and allows to define an alarm signal $S_{o}$ as follows

$$
S_{o}: \text { if } \quad \exists T / \eta(T)>\varepsilon_{o b s}
$$

2) Optical flow divergence (for avoidance): A weighted moving average function is used to keep a track of a reference optical flow. This allows the system acting on cam $_{f}$ to measure the variation of the optical flow when the rotorcraft moves laterally or upwards.

Taking into account $m$ measurements for the calculation of the moving average on the $k$ measurements from the beginning, the functions on $y$ and $z$ axes are is defined as follows

$$
\begin{aligned}
W M A_{O F_{y}^{f}} & =\frac{m \times\left(\bar{V}_{O F_{\mathrm{y}}}^{f}\right)_{k-m+1}+\ldots+\left(\bar{V}_{O F_{\mathrm{y}}}^{f}\right)_{k}}{\frac{m(m+1)}{2}} \\
W M A_{O F_{z}^{f}} & =\frac{m \times\left(\bar{V}_{O F_{\mathrm{z}}}^{f}\right)_{k-m+1}+\ldots+\left(\bar{V}_{O F_{\mathrm{z}}}^{f}\right)_{k}}{\frac{m(m+1)}{2}}
\end{aligned}
$$


Since the functions $\bar{V}_{O F_{\mathrm{y}}}^{f}(t), W M A_{O F_{y}^{f}}(t), \bar{V}_{O F_{\mathrm{z}}}^{f}(t)$ and $W M A_{O F_{z}^{f}}(t)$ depend on time, we can define the following alarm signals

$$
\begin{gathered}
S 1: \text { if } \forall t>0 \rightarrow \frac{d}{d t}\left(\bar{V}_{O F_{\mathrm{y}}}^{f}-W M A_{O F_{y}^{f}}\right)<0 \\
S 2: \text { if } \exists T \backslash\left\{\begin{array}{l}
\forall t<\left(T-T_{e}\right) \rightarrow \frac{d}{d t}\left(\bar{V}_{O F_{\mathrm{y}}}^{f}-W M A_{O F_{y}^{f}}\right)>0 \\
\forall t>\left(T+T_{e}\right) \rightarrow \frac{d}{d t}\left(\bar{V}_{O F_{\mathrm{y}}}^{f}-W M A_{O F_{y}^{f}}\right)<0
\end{array}\right. \\
S 3: \text { if } \quad \forall t>0 \rightarrow \frac{d}{d t}\left(\bar{V}_{O F_{\mathrm{z}}}^{f}-W M A_{O F_{z}^{f}}\right)<0
\end{gathered}
$$

where $S 1, S 2, S 3$ and $S 4$ are the alarm signals that indicate the lateral avoidance of an obstacle, the impossible lateral avoidance, the altitude avoidance and the impossible altitude avoidance, respectively.

\section{Dynamical Model}

The rotorcraft,with embedded cameras, that we use in our

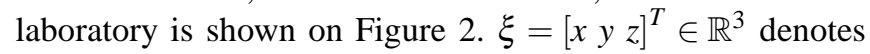
the position of the vehicle's center of gravity, relative to the inertial frame, and $\eta=\left[\begin{array}{lll}\phi & \theta & \psi\end{array}\right]^{T} \in \mathbb{R}^{3}$ are the three Euler angles (roll, pitch and yaw), which represent the orientation of the vehicle. The model of the full rotorcraft dynamics is obtained from Euler-Lagrange equations

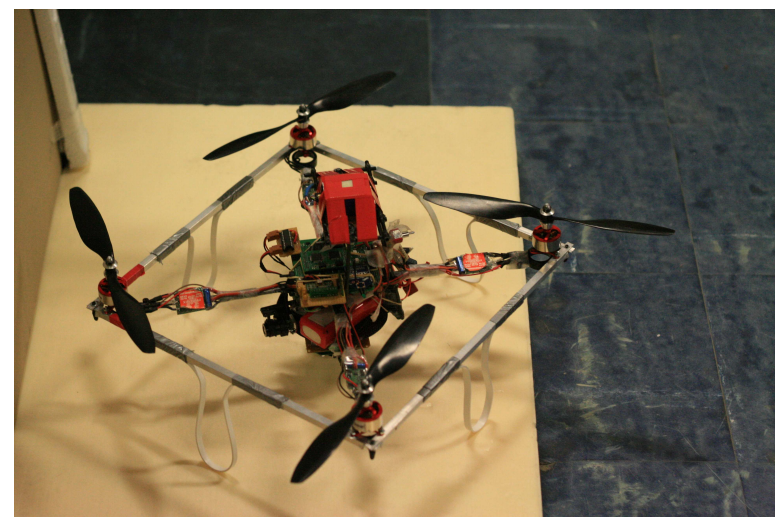

Fig. 2. Four rotor helicopter with cameras

$$
\begin{aligned}
m \ddot{x} & =-u \sin \theta \\
m \ddot{y} & =u \cos \theta \sin \phi \\
m \ddot{z} & =u \cos \theta \cos \phi-m g \\
m \ddot{\theta} & =\tilde{\tau}_{\theta} \\
m \ddot{\phi} & =\tilde{\tau}_{\phi} \\
m \ddot{\psi} & =\tilde{\tau}_{\psi}
\end{aligned}
$$

where $u, \tilde{\tau}_{\theta}, \tilde{\tau}_{\phi}, \tilde{\tau}_{\psi}$ are the force and torque inputs (see [1]).

\section{Control Strategy}

Because we aim to control the vehicle in speed and altitude, only equations (29), (30), (31) and (32) of the dynamical model are used. We take advantage of the fact that at a constant pitch angle, the vehicle moves in the forward direction at a constant speed. Similarly at a constant roll angle the vehicle is displaced at a constant velocity in the lateral direction.

\section{A. Low-Level Control}

The low-level control corresponds to the attitude control and the altitude control in order to stabilize the rotorcraft.

1) Attitude Control: Given equations (30), (31) and (32) we can identify three independent systems composed of two integrators in cascade. In order to assure the stabilization of such system we propose the following control strategy [14]

$$
\begin{aligned}
& \tilde{\tau}_{\theta}=-\sigma_{\theta_{1}}\left(K_{1}^{\theta}\left(\theta-\theta_{r e f}\right)\right)-\sigma_{\theta_{2}}\left(K_{2}^{\theta} \dot{\theta}\right) \\
& \tilde{\tau}_{\phi}=-\sigma_{\phi_{1}}\left(K_{1}^{\phi}\left(\phi-\phi_{r e f}\right)\right)-\sigma_{\phi_{2}}\left(K_{2}^{\phi} \dot{\phi}\right) \\
& \tilde{\tau}_{\psi}=-\sigma_{\psi_{1}}\left(K_{1}^{\psi} \psi\right)-\sigma_{\psi_{2}}\left(K_{2}^{\psi} \dot{\psi}\right)
\end{aligned}
$$

where $\sigma_{a}$ is a saturation function defined as

$$
\sigma_{a}(s)=\left\{\begin{array}{lc}
\mathrm{a} & s>\mathrm{a} \\
s & -\mathrm{a} \leq s \leq \mathrm{a} \\
-\mathrm{a} & s<-\mathrm{a}
\end{array}\right.
$$

2) Altitude Control: Once the attitude system is stabilized equation (29) becomes

$$
\ddot{z}=\frac{u}{m}-g
$$

Then, the control input $u$ is used to stabilize the altitude as follows

$$
u=m\left(-k_{1}^{z} \dot{z}-k_{2}^{z}\left(z-z_{r e f}\right)+g\right)
$$

which gives

$$
\ddot{z}=-k_{1}^{z} \dot{z}-k_{2}^{z}\left(z-z_{\text {ref }}\right)
$$

\section{B. Optical-flow-based Control}

1) Downward system: Let us recall the vehicle's speed sensed with respect to $\operatorname{cam}_{d}$ noted $\left(\bar{V}_{O F_{\mathrm{x}}}^{d}, \bar{V}_{O F_{\mathrm{y}}}^{d}\right)$. We use these two optical flows to design a speed controller which regulates the roll and pitch reference angles. The control strategy will be described only for the pitch angle, but we notice that the same control is implemented in the roll axis. Given $\bar{V}_{O F_{\mathrm{x}}}^{d}$ the output of the computing stage and $\bar{V}_{x}^{d}$ ref the desired optical flow reference, we define

$$
\theta_{r e f}=k_{p}^{\theta}\left(\bar{V}_{O F_{\mathrm{x}}}^{d}-\bar{V}_{O F_{\mathrm{x}}}^{d} r e f\right)+k_{i}^{\theta} \int_{0}^{T e}\left(\bar{V}_{O F_{\mathrm{x}}}^{d}-\bar{V}_{O F_{\mathrm{x}}}^{d} r e f\right) d t
$$

where $\theta_{\text {ref }}$ is the reference angle used in equation (33)and $T_{e}$ is the sampling time. By choosing the optical flow references values as variables, we can define three flying modes for the rotorcraft:

- Frontal displacement:

The vehicle moves in the pitch direction at a constant speed and at a constant altitude. $\bar{V}_{O F_{\mathrm{y}}}^{d}$ ref is set to zero. 
- Lateral displacement:

The vehicle moves in the roll direction at a constant speed and at a constant altitude. $\bar{V}_{O F_{\mathrm{x}}}^{d}$ ref is set to zero.

- Hover:

Here $\bar{V}_{O F_{\mathrm{x}}}^{d} r e f$ and $\bar{V}_{O F_{\mathrm{y}}}^{d} r e f$ are both set to zero to keep the rotorcraft stable in a constant position.

2) Frontal system: The rotorcraft's speed sensed with respect to $\operatorname{cam}_{f}$ is noted $\left(\bar{V}_{O F_{\mathrm{y}}}^{f}, \bar{V}_{O F_{\mathrm{z}}}^{f}\right)$. Also the time-tocontact with frontal obstacles is measured as in equation (19). The inverse of the time-to-contact is used to stop the vehicle when it is too close to obstacles, while the computed optical flow is used by the avoiding control strategy. We can then identify two subsystems: the detecting and the avoiding subsystem. First we detail the detecting subsystem for the quad-rotor flying in Frontal displacement mode. Given $\eta$ the inverse of the time-to-contact, we define the following proportional control

$$
\theta_{\text {prev }}=k_{\text {prev }}^{\theta} \eta
$$

where $\theta_{\text {prev }}$ is an angle of prevention, which is subtracted to the angle of reference $\theta_{\text {ref }}$ computed in equation (40). The gain $k_{p r e v}^{\theta}$ is choosen in a way that $\theta_{\text {ref }}$ becomes zero when $\eta$ is high enough to represent a imminent danger. Once the vehicle stops, an alert signal $S_{o}$ (as in (20)) is created to authorize the beginning of the avoiding sequence (avoiding controller). Next we describe the proposed control strategy for avoiding obstacle by lateral displacement. In a same way the controller is also implemented in the $z$ axis to allow altitude avoidance. Having the lateral optical flow $\bar{V}_{O F_{\mathrm{y}}}^{f}$ computed with $\mathrm{cam}_{f}$, and $W M A_{O F_{y}^{f}}$ as defined in equation (21) we propose the following control strategy

$$
\bar{V}_{a v} r e f=-k_{a v}^{\phi}\left\|\bar{V}_{O F_{\mathrm{y}}}^{f}-W M A_{O F_{y}^{f}}\right\|
$$

where $\bar{V}_{a v} r e f$ is a deceleration value applied to the reference optical flow $\bar{V}_{O F_{y}}$ ref on the lateral speed controller. Depending on which alarm signal is active (see section II-B.2), the rotorcraft knows if the obstacle has been crossed.

\section{State machine approach}

The overall system can be seen as a state machine associated with alarm signals and controllers depending on the environment analysis. The system always starts in Hover flying mode. When the user allows the displacement mode on the base station, the rotorcraft begins a Frontal displacement with the active obstacle detecting controller. The $S_{o}$ signal occurs when an obstacle is in front of the vehicle. It is important to note that when the $S_{o}$ signal is activated, the inverse of the time-to-contact $\eta$ is big enough to stop the vehicle. When this signal is activated the vehicle passes through the Lateral displacement flying mode with the active avoiding controller. The helicopter will stop automatically due to the avoiding controller, and it will check which alarm signal is active. If the signal $S_{1}$ is given, then the obstacle has been crossed and the Frontal displacement flying mode can restart again. In the opposite case, if the signal $S_{2}$ is given, then it is impossible to cross the obstacle on the lateral axis.
The Altitude displacement is then activated with the active avoidance controller. Similarly as before, the controller will automatically stop the rotorcraft, and check the active signal. If $S_{3}$ is active, then the obstacle has been crossed. However if the signal $S_{4}$ is given then a dead end is recognized and the helicopter stays in Hover flying mode until the base station allows the stopping sequence.

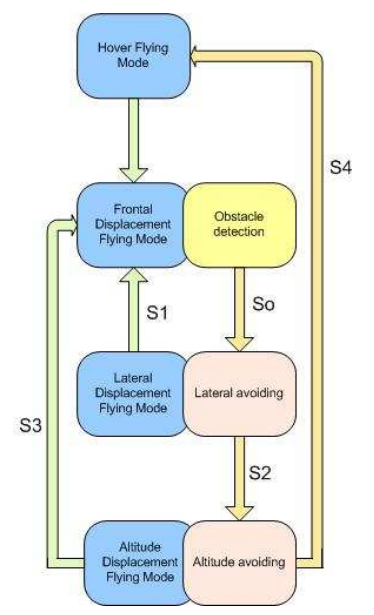

Fig. 3. Overall state machine system

\section{EXPERIMENTAL APPLICATION}

The real-time implementation of such a strategy on our four rotor rotorcraft is quite difficult since the use of two cameras needs appropriate calculations on either on board microprocessors which require high memory (and which is a difficult task) or on a ground station. In the second case, images have to be transmitted from embedded cameras to the ground computer and videos transmissions suffer from delays and information losses. In order to validate our algorithm, we have decided to test each stage independently and firstly in a mobile robot platform. Two orthogonal cameras have been mounted into a remote control car (see Figure 4). One of the camera is pointing forward when the other is pointing towards the left side of the car. This configuration copies exactly the system described for the four rotor helicopter in the sense that the left side camera behaves like the downward camera in the helicopter. Our goal is to validate the different optical-flow-based controllers. The first test has been conducted to control the forward velocity of the robot to a constant set point without the anti-collision system. The second test was carried with the detecting controller. The first experiment starts at rest (no initial velocity) and shows that with the only optical flow measurement the controller can accelerate the robot until the velocity set point is reached. When this velocity has been reached, it has to remain the most constant possible. Since the cameras have not been calibrated (there is no need to calibrate them in this experiment) and since we really don't know the depth of the scene, the control is done in the image coordinate system. As shown in Figure 5, the controller achieves, as expected, the control 


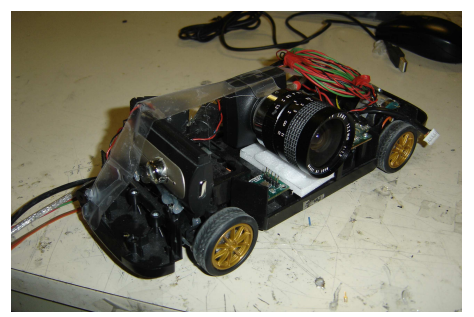

Fig. 4. Camera setup in the car

of the robot velocity. Only small changes in the optical flow are noticed, but they do not represent great discontinuities that could damage the control strategy. The anti-collision

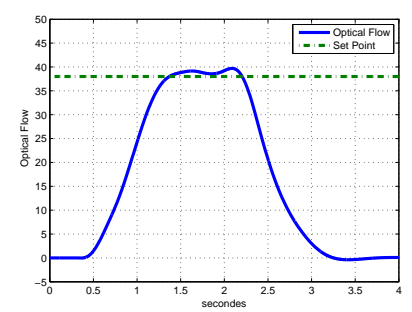

Fig. 5. Speed control results

controller has been tested on the same platform, and under the same conditions. An obstacle has been introduced in the front of the mobile robot. Different tunings of the controller have been tested and different distances of stops have been achieved. This result is also expected and desired, because it means that we can easily change the interval of confidence in between an obstacle is or is not dangerous. The signal of transition has been simulated by maintaining the inverse of the time-to-contact equal to the threshold value that stops the vehicle (Figure 6). We noticed that there exists a small delay between the time-to-contact and the velocity estimation due to the filtering sequences of the optical flow used for the speed control. We remark that the time-to-contact has not the same signification when the forward velocity is not constant. Therefore, the lateral (or downward) camera is very useful in order to maintain a constant velocity and also to have a good understanding of the time-to-contact.

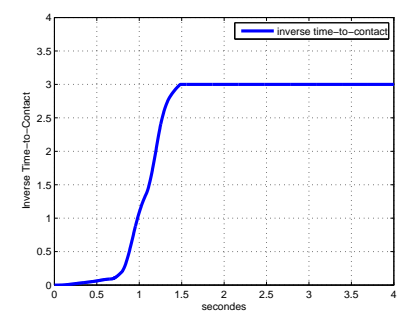

Fig. 6. Coupling link between speed control and time-to-contact

\section{CONCLUDING REMARKS}

In this work we present a reactive navigation system for an unmanned rotorcraft using only the optical flow of two orthogonal cameras, and the measurements of an inertial measurement unit. We show that most of the information given by the optical flow can only be wisely used if the speed of travel is constant. In order to maintain the speed of travel equal to a constant set point, a first controller was built and tested on a mobile robot. The speed controller has been completed with a detection and avoidance system which uses a signal alarm approach in order to commute between different flying modes. The proposed method used the capability of hovering flight to force a continuity between each control law. We are currently implementing the first step controller on a four rotor helicopter and as future work we intend to complete the system with wall following and centering behavior as principal states, taking advantage of the constant forward motion.

\section{REFERENCES}

[1] F. Kendoul, D. Lara, I. Fantoni-Coichot, and R. Lozano, "Real-time nonlinear embedded control for an autonomous quadrotor helicopter," AIAA, Journal of Guidance, Control, and Dynamics, vol. 30, no. 4, pp. 1049-1061, July-August 2007.

[2] S. Salazar, J. Escareno, D. Lara, and R. Lozano, "Embedded control system for a four rotor UAV," International Journal of Adaptive Control and Signal Processing, vol. 21, no. 2-3, pp. 189-204, MarchApril 2007.

[3] S. Hrabar, G. S.Sukhatme, P. Corke, K. Usher, and J. Roberts, "Combined optic-flow and stereo-based navigation of urban canyons for a UAV," in International Conference on Intelligent Robots and Systems, vol. 3, no. 4, Alberta,Canada, 2005, pp. 198-209.

[4] T. Camus, D. Coombs, M. Herman, and T.-H. Hong, "Real-time singleworkstation obstacle avoidance using only wide-field flow divergence," Videre: Journal of Computer Vision Research, vol. 1, no. 3, pp. 30-57, 1999.

[5] H. Romero, S. Salazar, A. Sanchez, and R. Lozano, "A new UAV configuration having eight rotors : Dynamical model and real-time control," in IEEE Conference on Decision and Control, New Orleans,USA, 2007.

[6] B. Herisse, F. Russotto, T. Hamel, and R. Mahony, "Hovering flight and vertical landing control of a VTOL unmanned aerial vehicle using optical flow," in IEEE/RSJ International Conference on Intelligent Robots and Systems, Nice, France, 2008.

[7] J. Serres, F. Ruffier, S. Viollet, and N. Franceschini, "Toward optic flow regulation for wall-following and centring behaviours," International Journal of Advanced Robotic Sytems, vol. 3, no. 2, pp. 147-154, 2006.

[8] J.-C. Zufferey and D. Floreano, "Toward 30-gram autonomous indoor aircraft:vision-based obstacle avoidance and altitude control," in IEEE International Conference on Robotics and Automation, Barcelona, Spain, 2005.

[9] J.-C. Zufferey, A. Klaptocz, A. Beyeler, J.-D. Nicoud, and D. Floreano, "A 10 gram microflyer for vision-based indoor navigation," in IEEE International Conference on Intelligent Robots and Systems, Beijing,China, 2006.

[10] J.-Y. Bouguet, "Pyramidal implementation of the Lucas Kanade feature tracker," Intel Corporation, Microprocessor Research Labs, Technical report, Tech. Rep., 1999.

[11] H. Romero, S. Salazar, R. Lozano, and R. Benosman, "Fusion of optical flow and inertial sensors for four rotor rotorcraft stabilization," in 6th Symposium IFAC on Intelligent Autonomous Vehicles (IAV 2007), Toulouse, France, 2007.

[12] L. Muratet, S. Doncieux, Y. Brière, and J.-A. Meyer, "A contribution to vision-based autonomous helicopter flight in urban environments," Robotics and Autonomous Systems, vol. 50, no. 4, pp. 195-209, 2005.

[13] S. Umeyama, "Least-squares estimation of transformation parameters between two point patterns," IEEE Trans. on Pattern Analysis and Machine Intelligence, vol. 13, no. 4, pp. 376-380, 1991.

[14] S. Salazar, H. Romero, R. Lozano, and P. Castillo, "Modeling and real-time stabilization of an aircraft having eight rotors," Journal of Intelligent and Robotic Systems, vol. 54, pp. 455-470, 2008. 